\title{
Positioning two Redundant Arms for Cooperative Manipulation of Objects
}

\author{
Adrià Colomé and Carme Torras
}

\begin{abstract}
Bimanual manipulation of objects is receiving a lot of attention nowadays, but there is few literature addressing the design of the arms configuration. In this paper, we propose a way to analyze the relative positioning of two redundant arms, both equipped with spherical wrists, in order to obtain the best common workspace for grasping purposes. Considering the geometry of a robot with a spherical wrist, the Cartesian workspace can be discretized, with an easy representation of the feasible end-effector orientations at each point using bounding cones. After having characterized the workspace for one robot arm, we can evaluate how good each of the discretized poses relate with an identical arm in another position with a quality function that considers orientations. In the end, we obtain a quality value for each relative position of two arms, and we perform an optimization using genetic algorithms to obtain the best workspace for a cooperative task.
\end{abstract}

Key words: Robot Design, Workspace, Bimanual Manipulation.

\section{Introduction}

Bimanual manipulation allows robots to perform more complex tasks than a singlelimb robot [1]. However, while a lot of attention is focused on how to manipulate or plan a task, less importance is given to the arms configuration. Usually, a humanoidlike configuration is chosen, to make the robot more human-friendly [2], but when deciding how to use two Barrett WAM (Whole Arm Manipulator) robots, we questioned ourselves if a humanoid-like configuration would be the best, for example, for folding clothes. A first step towards this aim is to analyze the robot's workspace. In [3], a discretized workspace is used with information about the probability of

This work is partially supported by EU Project IntellAct (FP7-269959), by the CSIC Project CINNOVA (201150E088), and by the Catalan Research Commission through SGR-00155.

Adrià Colomé · Carme Torras

Institut de Robòtica i Informàtica Industrial (CSIC-UPC), e-mail: [acolome,torras]@iri.upc.edu 
solving the Inverse Kinematics (IK) with random orientations at each cartesian position, and also manipulability data, an indicator of dexterity and distance to a singularity [4], in order to decide the grasping points for bimanual manipulation. However, this work exploits an existing humanoid robot, which may not have been specifically designed for the task being tackled. Zacharias et al. [5, 6, 7] plot the $3 D$ cartesian position workspace by initially drawing spheres, whose color varies with the percentage of inverse kinematics solutions found for each point. Moreover, they propose to use different shapes at each point to represent orientations, depending on the feasible end-effector orientations at each position, but their later work also focuses on optimizing manipulation with a given bimanual robot, rather than deciding its arms configuration.

We think that the relative position of the arms may be improved in order to get better grasps. In fact, the WAM robot's workspace is much different from that of a human arm, and so are those of many other commercial arms, thus we investigated a way of deciding the arms relative position, depending on the task, by fully characterizing the workspace of the Barrett WAM arm. Using the fact that the robot has a spherical wrist, we propose to compute its feasible orientations for each cartesian point and pack them in a bounding cone to obtain an easy characterization of robot feasible poses. With these cones, we can evaluate how good two points relate when grasping an object of a certain length. If both robots can reach the position, but their valid end-effectors' orientations for those points are not good enough, the object may not be well manipulated. In Section 2 we will explain how we characterize the workspace with information on all feasible orientations and how we store these data. This is later used in Section 3 to evaluate a relative positioning of two identical arms. Finally, in Section 4 we describe the implementation, and we use a genetic algorithm to search for the best relative positioning of the two arms.

\section{Workspace Representation}

For a redundant robot, it is well known that the Forward Kinematics (FK) function $f$ is not one-to-one, and given its non-linearity, the workspace may be hard to represent. The Inverse Kinematics (IK), the inverse of the FK, may then be better to characterize the workspace. Note that, for each point in the cartesian space, more than one IK solution may exist [8]. We initially decided to characterize the workspace numerically, as a subset of $\mathbb{R}^{3} \times S O(3)$, by discretizing it. To do so, a uniform mesh is set for the cartesian position and/or orientation, and, for each point $P_{i}$ on the mesh, the existence of a joint solution $\theta^{P_{i}}$ such that $P_{i}=f\left(\theta^{P_{i}}\right)$ is checked. This can be done by sampling the joint space and using the FK function (forward sampling), or by sampling the workspace and using the IK (inverse sampling). Nevertheless, while the forward sampling results in a biased sampling of the workspace, the inverse is able to exhaustively analyze the whole workspace, thus we recommend this option if a good IK algorithm is available (for the case of the WAM robot, the IK can be obtained either by iterative methods [8] or analytical methods [9, 10]).

To plot the reachable positions of the workspace, and store its data, we used a similar method as in [7]. For each point of the $3 D$ mesh representing the workspace, $M$ solutions of the IK of the robot, with different orientations, are obtained. To 
ensure a good distribution of these orientations, we can use the proposal in [12], where points are arranged in hexagonal patterns to fit on the sphere, or use randomly generated quaternions. If there exists at least one solution, the position is reachable. In addition, for each of these $M$ IK attempts, we can extract additional information, such as manipulability [4] at the obtained pose, percentage of orientations found for a given $3 D$ cartesian point, etc.

For the feasible orientations of a robot arm in a cartesian point, several geometrical shapes to represent the valid orientations have been proposed in literature [7]. Among these shapes, cones are probably the best choice, due to their simplicity and easy characterization. In fact, for a robot with a spherical wrist (see Fig. 1a), the Tool Center Point (TCP) stays within a cone whose axis is the rotation axis of the first degree of freedom of the wrist (namely, the forearm axis).

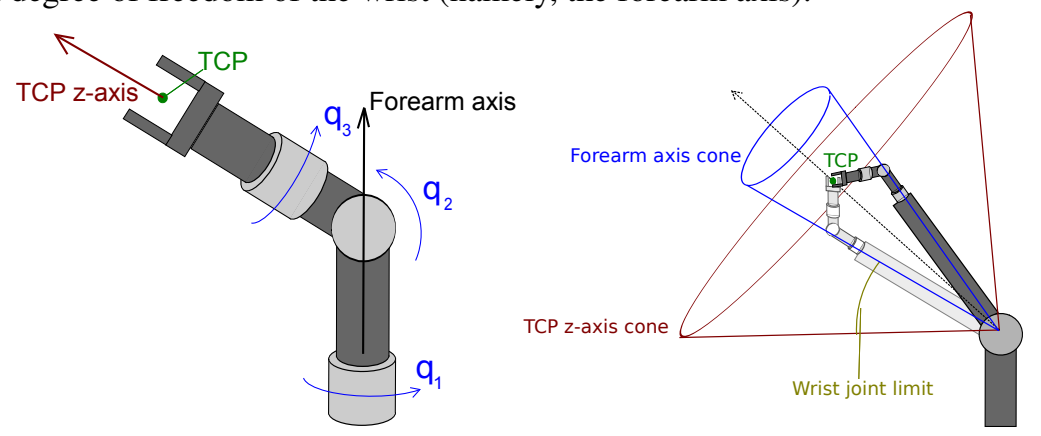

Fig. 1: Left: Spherical wrist. $\theta_{2}$ is the wrist angle. Right: Robot scheme showing the bounding cone of all possible forearm axes. This cone is augmented by adding the wrist joint limit to obtain the possible TCP $z$-axis bounding cone.

Moreover, discarding the rotation around the TCP $z$-axis, we propose to collect the set of valid forearm axes at a certain cartesian point $P \in \mathbb{R}^{3}$, which will be enclosed by a cone, and compute the Bounding Cone (BC) that contains them all with the algoritm proposed in [11]. Also, if the wrist angle has symmetric limits, its aperture can be added to the $\mathrm{BC}$ angle, yielding a cone that contains all the TCP z-orientation axis that the robot can reach at the given position (see Fig. 1b).

With this approach, we obtain a mesh for the workspace, encoding all the information gathered when computing the reachable positions such as manipulability, percentage of solutions found, etc. plus the obtained bounding cone containing all the possible $z$-axis of the TCP. We can see an IK solutions map over the workspace of a WAM robot (see Table 1 in [10] for its dimensional parameters) in Fig. 2.

\section{Bimanual Workspace}

Multiple-arm cooperative tasks provide the capability of performing tasks that would be impossible or, at least, much more difficult to accomplish with only one arm. Although actuating the arms to simultaneously move an object may be a hard task, the arms relative configuration must be given importance, since depending on the intended use of bimanual robots, some configurations might be better suited 


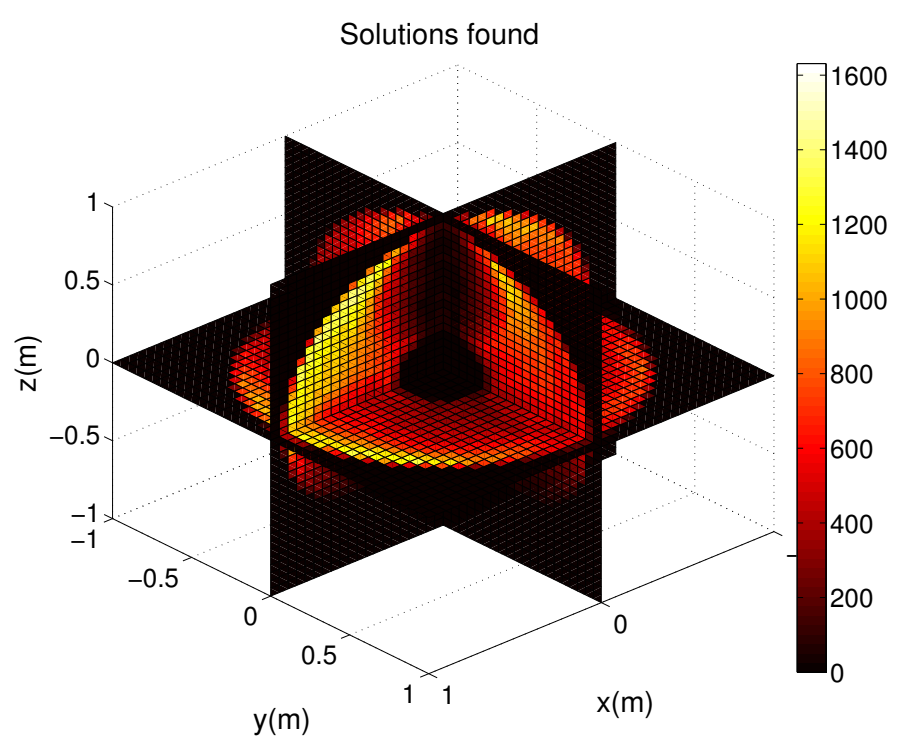

Fig. 2: Solutions found over the workspace of a WAM robot.

than others. Human arms configuration may be the best for the tasks performed by humans along their evolution, with a large workspace in front, and a very reduced workspace at our back, as our attention and visible space stays in front of us.

In [1], a review of bimanual manipulation is done, where the state-of-the-art in cooperative tasks is analyzed. Some examples of existing bimanual robots are shown, such as the Justin robot [6], where the arms are placed in a humanoid-like configuration with a tilt of 60 degrees, the DARPA arm robot [13], with two Barrett WAM robot arms with their bases placed perpendicular, or the ARMAR [14] [15] robot, where both $z$ base axes are placed in an aligned humanoid-like configuration.

However, the humanoid configuration may not be the best one for certain tasks. In this section, we provide hints to determine how good is a relative positioning of robot arms, in the form of a parametrized value function to be used with a numerically characterized workspace as that in the previous section.

\subsection{Proposed Quality Function}

In this work, we intend to characterize a common workspace between two arms. To this purpose, given two cartesian points $P_{1} \in W_{1}, P_{2} \in W_{2}$, we will compute several factors that will lead to a quality function for each pair $\left(P_{1}, P_{2}\right)$ defined as:.

$$
F\left(P_{1}, P_{2}\right)=D F \cdot S D F \cdot O F \cdot M F \cdot C F,
$$

where $D F$ is the Distance Factor, $S D F$ the Solutions Density Factor, $O F$ the Orientation Factor, $M F$ the Manipulability Factor and $C F$ the Conditioning Factor. Mul- 
tiplication and not addition of factors has been chosen to strongly penalize those positions with very low value on one factor.

Then, the global quality value of a relative position of two arms is:

$$
F=V \cdot \sum_{P_{1} \in W_{1}} \sum_{P_{2} \in W_{2}} F\left(P_{1}, P_{2}\right),
$$

$V$ being the total volume of the combined workspace. Evaluating this quality measure (1) we get a mapping $g: \mathbb{R}^{3} \times S O(3) \rightarrow \mathbb{R}$, which maps a relative position plus orientation transformation (up to 6 variables) to a real value. This mapping can be used by genetic algorithms to search for its maximum, which would correspond to the best relative positioning.

\subsubsection{Points to Compare and Distance Factor}

For each pair of points $\left(P_{1}, P_{2}\right)$, we have to decide whether to evaluate their relation or not. In order to decide that, we may take a characteristic length $L$ for the object to be manipulated, and then one possible way to evaluate that relation is by using the following Distance Factor (DF):

$$
D F=\left\{\begin{array}{l}
1 \text { if } L-\delta<\left\|P_{2}-P_{1}\right\|<L+\delta,\left|a_{1}\right|<\alpha_{1}, \text { and }\left|a_{2}\right|<\alpha_{2}, \\
0 \text { otherwise }
\end{array}\right.
$$

where $\delta$ is a tolerance on the manipulated object length, and $a_{1}, a_{2}, \alpha_{1}, \alpha_{2}$ are defined with the bounding cones in Fig. 3. If the segment joining $P_{1}$ and $P_{2}$ does not lie within both orientation cones for $P_{1}$ and $P_{2}$, their relation may not be evaluated. However, the orientation restriction can be made more permissive, depending on the kind of graspings to perform.

\subsubsection{Solution Density Factor}

As defined in some previous works [3,7], the SDF is the ratio of the IK solutions found over the attempted solutions. For each cartesian point of the workspace, we retain the percentage of IK solutions found, given random orientations. The SDF is then defined as the product of the ratios for the two points compared.

\subsubsection{Orientation Factor}

Imagine two arms manipulating an object of length $L$ grasped at points $P_{1} \in W_{1}$ and $P_{2} \in W_{2}$. In this situation, a grasp in which the TCP $z$-axis of each arm is aligned in the direction of the other grasping point is usually preferred. This can be checked using the cones $\left(Z_{1}, \alpha_{1}\right),\left(Z_{2}, \alpha_{2}\right)$ obtained for each workspace: we can calculate the angles $a_{1}, a_{2}$ from the vector $P_{1}-P_{2}$ and the cones axes $Z_{1}, Z_{2}$, as in Fig. 3.

Then, we define the Orientation Factor $(O F)$ for the points to compare as

$$
O F=\max \left(O F_{\min }, 1+\frac{1}{K} \ln \left(\frac{\alpha_{1}-\left|a_{1}\right|}{\alpha_{1}} \cdot \frac{\alpha_{2}-\left|a_{2}\right|}{\alpha_{2}}\right)\right)
$$

where $K$ is a tuning parameter, and $O F_{\text {min }}$ is the minimum value accepted for the orientation factor. 


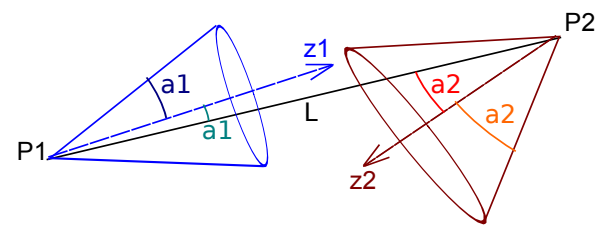

Fig. 3: Distance and Orientation factor variables: $\alpha_{1}, \alpha_{2}$ are the cones angles, and $a_{1}, a_{2}$ the angles between each cone's axis and the line $P_{1} P_{2}$.

Thus defined, this factor verifies that $O F_{\min } \leq O F \leq 1, \forall a_{1}, a_{2}, \alpha_{1}, \alpha_{2}$, when satisfying the conditions in (2), it having a value of 1 when both cones' axes are parallel to the vector $P_{2}-P_{1}$ and pointing towards each other, and gradually reducing its value to $O F_{\min }$ when the axes point away from each other.

\subsubsection{Manipulability Factor}

When performing cooperative tasks, or grasping an object with multiple arms, there are approaches to obtain a combined manipulability [16, 17]. However, combined manipulability computation for multiple arms holding an object relies on the arms poses, which are unknown for our workspace representation, as we use the average of many IK computations with different orientations and the redundancy of the robot gives us infinite solutions. So we take the average manipulability of both grasping points as a good approach to evaluate how manipulable is an object. In [3], grasping point candidates are selected based on this manipulability, so the $M F$ is defined as:

$$
M F\left(P_{1}, P_{2}\right)=\bar{m}\left(P_{1}\right) \bar{m}\left(P_{2}\right), \text { with } \bar{m}\left(P_{i}\right)=\frac{1}{M} \sum_{j=1}^{M} m\left(I K\left(P_{i}, o_{j}\right)\right),
$$

$m\left(I K\left(P_{i}, o_{j}\right)\right)$ being the manipulability at the joint position obtained as an IK solution for the robot at position $P_{i}$ with orientation $o_{j}$.

\subsubsection{Conditioning Factor}

In order to ensure a stable behaviour for the related points, we use the Jacobian Condition Number $(\mathrm{CN})$, which is defined as $\kappa(J)=\sigma_{1} / \sigma_{n}$, where $\sigma_{1}, \sigma_{n}$ are the largest and the smallest singular values of the robot Jacobian matrix. The $\mathrm{CN}$ is a measure of the error amplification induced by the Jacobian matrix. Thus, we define

$$
C F=\kappa\left(P_{1}\right) \cdot \kappa\left(P_{2}\right)
$$

where $\kappa\left(P_{i}\right)$ is the average $\mathrm{CN}$ for the solved IK of $P_{i}$.

\section{Experimentation}

As a first application, we searched for optimal relative positionings of two WAM robots. To do so, we used genetic algorithms instead of performing an exhaustive analysis in order to obtain the results faster, using 10 generations of 20 elements each, and a probability of mutation on each variable slightly decreasing after each generation. 
We considered valid objects for grasping those of a size between $0.3 \mathrm{~m}$ and $0.5 \mathrm{~m}$ for the DF and a $K=2$ for the OF. We collected the best half at each generation, paired them, and created 20 new configurations for the next generation.

Several settings were considered for optimizing a two-dimensional relative position between the arms. The first one is similar to that of the DARPA robot, with both $z$ axes perpendicular. We found (see Fig. 4 top) that, for our criterion, the best configurations are those with positive $d x$ and $d z$, while the DARPA robot has both negative offsets, which yield a lower value of the quality function. The best solution is for $d x=0.8 m, d z=0.8 \mathrm{~m}$ in which both arms cooperate at a larger distance than the DARPA robot. Other experimentation such as placing both arms with their $z$-axes parallel and facing each other (see Fig. 4 bottom) also lead to good positionings which might not have been considered when building bimanual robots.
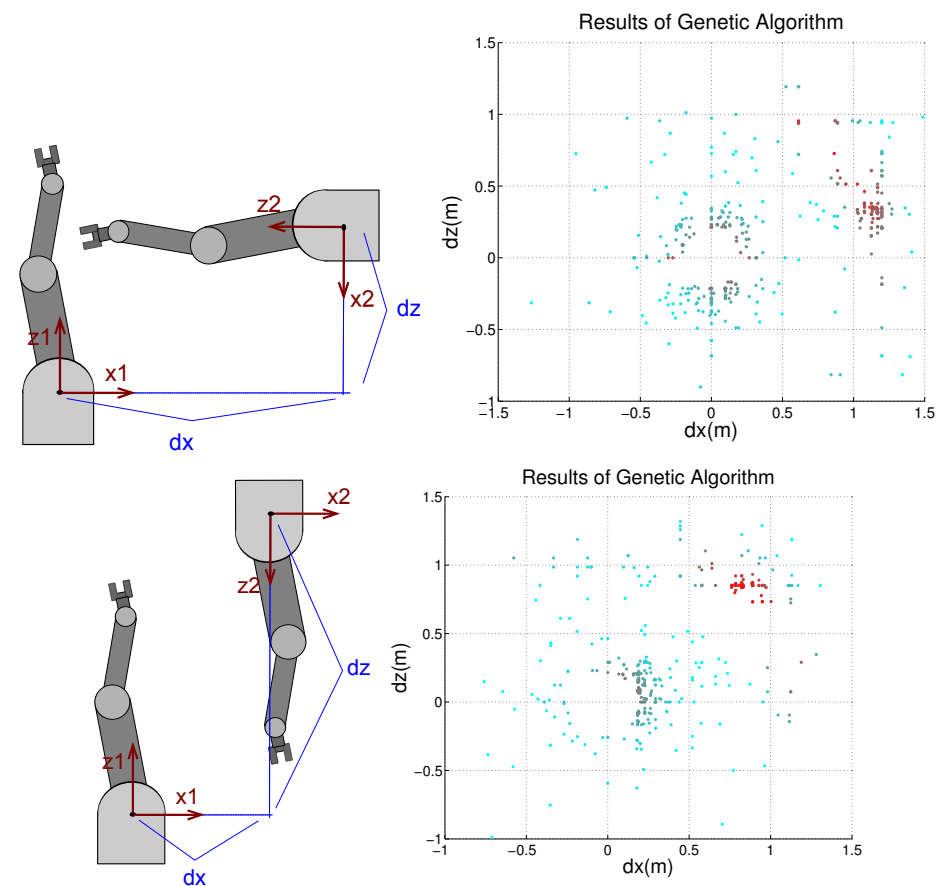

Fig. 4: Left: Two experimental settings. Right: Results with quality values from blue (low value) to red (high value).

\section{Conclusions}

In this work, we have presented a novel way to store the workspace information (including orientation, which is often not considered) of a robot with a spherical wrist, in a very compact way, thanks to the efficient bounding cones representation of the end-effector $z$-axis. This then allows us to evaluate the capability of a dual arm robot to manipulate an object of a certain size, depending on the relative position of both arms. We can compute a global quality measure for a given relative position, in order to quantify how good a dual arm configuration is. 
We used this quality measure to obtain better relative configurations with the help of a genetic algorithm. And the results in Section 4 seem to indicate that, for the tasks studied, the configuration of current bimanual robots may not be optimal.

The arm configurations of humanoid robots are designed to accomplish a wide repertoire of tasks while obeying diverse design and operational constraints. However, in settings with two independent robot arms, it may be simple and advantageous to tailor their relative configuration to the specificities of each particular task, as shown in the current work. The proposed algorithm can be further used to optimize relative positionings with more than two parameters in order to get more general results.

\section{References}

1. C. Smith, Y. Karayiannidis, L. Nalpantidis, X. Gratal, P. Qi, D.V. Dimarogonas, D. Kragic, "Dual arm manipulation-A survey". Rob. and Autonomous Syst, no. 60, pp 1340-1353, 2012.

2. S.-H. Hyon,J. G. Hale and G. Cheng, "Full-Body Compliant Human-Humanoid Interaction:Balancing in the Presence of Unknown External Forces" IEEE Trans. on Robotics, vol 23 , no. 5, 2007.

3. N. Vahrenkamp, M. Przybylski, T Asfour, R. Dillmann, "Bimanual Grasp Planning" IEEERAS Int. Conf. on Humanoid Robots, pp 493-499, 2011.

4. T. Yoshikawa. "Dynamic manipulability of robot manipulators" IEEE ICRA, vol 2, pp 1033$1038,1985$.

5. F. Zacharias, D. Leidner, F. Schmidt, C. Borst, G. Hirzinger. "Exploiting Structure in Twoarmed Manipulation Tasks for Humanoid Robots" IEEE/RSJ IROS, pp 5446-5452, 2010.

6. Ch. Ott, O. Eiberger, W. Friedl, B. Bauml, U, Hillenbrand, Ch. Borst, A. Albu-Schaffer, B. Brunner, H. Hirschmoller, S. Kielhofer, R. Konietschke, M. Suppa, T. Wimbock, F. Zacharias, G. Hirzinger. "A Humanoid Two-Arm System for Dexterous Manipulation" IEEE RAS Conf. on Humanoid Robots, pp 276-283, 2006.

7. F. Zacharias, C. Borst, G. Hirzinger, "Capturing Robot Workspace Structure: Representing Robot Capabilities" IEEE/RSJ IROS, pp 3229 - 3236, 2007.

8. A. Colomé and C. Torras. "Redundant inverse kinematics: Experimental comparative review and two enhancements" IEEE/RSJ IROS, pp. 5333-5340, 2012.

9. M. Shimizu, H. Kakuya, W.-K. Yoon, K. Kitagaki, and K. Kosuge. "Analytical inverse kinematic computation for 7-dof redundant manipulators with joint limits and its application to redundancy resolution" IEEE Trans. on Robotics, pp 1131-1142, 2008.

10. G. K. Singh, J. Claassens, "An Analytical Solution for the Inverse Kinematics of a Redundant 7-dof Manipulator with Link Offsets" IEEE/RSJ IROS, pp 2976-2982, 2010.

11. G. Barequet, G. Elber. "Optimal Bounding Cones of Vectors in Three Dimensions" Information Processing Letters, vol. 93 no. 2, pp 83-89, 2005.

12. E. Saff, A. Kuijlaars, "Distributing many points on the sphere" Mathematical Intelligencer, vol. 19, no. 1, pp 5-11, 1997.

13. E. Guizzo, "DARPA Seeking to Revolutionize Robotic Manipulation" IEEE Spectrum: Technology and Science News, url: http://spectrum.ieee.org/automaton/robotics/roboticssoftware/darpa-arm-program , 2010.

14. T. Asfour, K. Berns, R. Dillmann, "The humanoid robot ARMAR: Design and control" IEEE/APS Int. Conf. on Humanoid Robots, pp 7-8, 2000.

15. K. Regenstein,P. Azad, J. Schroder,A. Bierbaum,N. Vahrenkamp, R. Dillmann, ”ARMARIII: An Integrated Humanoid Platform for Sensory-Motor Control" IEEE/RAS Int. Conf. on Humanoid Robots, pp 169-175, 2006.

16. P. Chiacchio, S. Chiaverini, L. Sciavicco, B. Siciliano. "Global Task Space Manipulability Ellipsoids for Multiple-Arm Systems" IEEE Trans. on Rob. and Aut., vol. 7, no. 5, 1991.

17. A. Bicchi, D. Prattichizzo. "Manipulability of Cooperating Robots with Unactuated Joints and Closed-Chain Mechanisms" IEEE Trans. on Rob. and Aut., vol. 16, no. 4, 2000. 